\title{
Identidad patrimonial, emociones $y$ enseñanza de las Ciencias Experimentales
}

\section{Heritage identity, emotions and teaching of Experimental Sciences}

DOI: $10.7203 / D C E S .38 .15688$

\author{
Bartolomé Vázquez-Bernal \\ Universidad de Huelva, bartolome.vazquez@ddcc.uhu.es \\ ORCID iD: https://orcid.org/0000-0002-9120-5751 \\ M. Ángeles de las Heras Pérez \\ Universidadde Huelva, angeles.delasheras@ddcc.uhu.es \\ ORCID iD: https://orcid.org/0000-0002-3640-8337 \\ Roque Jiménez-Pérez \\ Universidad de Huelva, rjimenez@ddcc.uhu.es \\ ORCID iD: https://orcid.org/0000-0001-7779-0061
}

\begin{abstract}
RESUMEN: En el presente trabajo se presenta parte de una secuencia de indagación en el aula de Química para el estudio de la materia, en concreto, una salida de aula con la intención de trabajar aspectos relacionados con los métodos de separación de las mezclas a partir de un elemento patrimonial como es un molino de mareas (Zaporito). Se pretende conocer qué cambios experimentan las emociones del alumnado hacia esta actividad medidas antes de la misma, durante y después; poner de manifiesto, si es posible, el vínculo ciencias experimentales y educación patrimonial, así como decidir entre beneficios y costes de la experiencia. Se observa cómo las emociones positivas están presentes en los tres momentos del estudio por encima de las emociones negativas. Se pone de manifiesto cómo es posible el trabajo de las ciencias experimentales a través de elementos patrimoniales, lo que supone una ruptura con la incomunicación entre éstas y las ciencias sociales. Además, la experiencia ayudó a la toma de decisiones.
\end{abstract}

Palabras Clave: emociones, patrimonio, educación secundaria obligatoria, enseñanza de la química, indagación.

ABSTRACT: This paper presents part of an inquiry sequence in the Chemistry classroom for the study of matter, specifically, a field trip with the intention of working on aspects related to the separation methods of mixtures from a heritage element such as a tidal mill (Zaporito). The aim is to find out the changes experienced in students' emotions towards this activity, measured before, during and after it; to show, if possible, the link between experimental sciences and heritage education, as well as to decide between benefits and costs of the experience. It is observed how positive emotions are present in the three moments over negative emotions. It is shown how the work of the experimental sciences is possible through heritage elements, which means a break with the lack of communication between these and the social sciences. Besides, the experience helped decision making.

KEYWORDS: emotions, heritage, compulsory secondary education, chemistry teaching, inquiry.

Fecha de recepción: julio de 2019

Fecha de aceptación: enero de 2020

Financiación a través del Proyecto de Educación Patrimonial para la Inteligencia Territorial y Emocional de la Ciudadanía (EPITEC) (EDU2015-67953-P). 


\section{ANTECEDENTES: LA CIENCIA COMO CULTURA}

Existe un consenso generalizado desde la Didáctica de las Ciencias Experimentales, en entender la ciencia como parte de la cultura (Sánchez-Mora, 2013). La ciencia está asistida por una construcción humana y es caudal de la cultura de la sociedad (UNESCO-Montevideo, 1999). Contrapuesta a una imagen de la ciencia para una élite, aislada por la investigación disciplinar e inalcanzable para la ciudadanía (reforzado en los materiales escolares), existe otro concepto de ciencia social, cercana y de utilidad para la vida (Manassero, Vázquez y Acevedo, 2002), que es clave para la idea de alfabetización científica y tecnológica necesaria para la toma de decisiones ante cuestiones sociocientíficas en un debate argumentado críticamente (Solbes, 2013), un valor intrínseco de las sociedades democráticas (Retana-Alvarado, Vázquez-Bernal y Camacho, 2018).

Así mismo, Tobin (2010) entiende el conocimiento como una representación cultural, es decir, que el aprendizaje de las ciencias es una representación de la cultura científica. Sin embargo, sigue imperando la idea en ciertos sectores de una ciencia escolar propedéutica (Osborne, Simon y Collins, 2003), amparada por el propio sistema y los gestores del currículo oficial.

Pero además, en su caso, no es suficiente que el profesorado sepa que el conocimiento científico está incrustado en la sociedad y la cultura, sino que debe ser capaz de usar episodios actuales e históricos simplificados de la práctica científica y tecnológica (Acevedo Díaz, García-Carmona y Aragón, 2016), que ilustren la Naturaleza de las Ciencias como parte fundamental de la alfabetización científica cultural (Showalter, 1974; citado por Lederman, 2018) y su incidencia en la ciudadanía (Hodson 2014). Lamentablemente, su presencia es mejorable en la enseñanza de las ciencias de los diferentes niveles educativos (Acevedo Díaz, García-Carmona y Aragón, 2016). En este trabajo se analiza una propuesta de aula, en consonancia con lo anterior, centrándose en elementos emocionales, patrimoniales y científicos.

\section{MARCO TEÓRICO}

\subsection{El patrimonio cultural y la interdisciplinariedad}

Actualmente cada vez es más aceptada la interdependencia entre el conocimiento científico y los factores sociales que los delimitan y los controlan, siendo usual la caracterización de la sociedad del conocimiento como la expresión simbiótica entre ciencia y sociedad de consumo, en los sectores industriales y culturales, englobados como sectores económicos. El patrimonio cultural ha evolucionado conceptualmente desde el siglo XIX, más allá de lo histórico-artístico, realzando en la legislación educativa su carácter holístico, simbólico y de identidad de los pueblos (Castro-Martínez, 2018). Sin embargo, en los libros de textos continúa la visión academicista, disciplinar, y monumentalista (Cuenca, Estepa-Giménez y Martín-Cáceres, 2017), además de otras expresiones culturales, que deben ser consideradas para su conocimiento, conservación, difusión y utilidad como es el patrimonio tecnológico, considerado el nexo de unión entre la didáctica de las ciencias experimentales, hoy desarrollada a través del movimiento C-T-S con la didáctica de las ciencias sociales, mediante la nueva corriente de conceptualización del patrimonio (Cuenca, 2002). Esta interdisciplinariedad concibe la situación mucho más real para el entendimiento de la naturaleza de las ciencias y la importancia de la ciencia en la cultura.

La concepción de patrimonio desde la cual partimos en el presente estudio (Jiménez-Pérez, Cuenca y Ferreras, 2010; Cuenca, Estepa-Giménez y Martín Cáceres, 2017), se entiende desde una visión amplia e integral, con una perspectiva holística, definiendo como patrimonio la conjunción de elementos artísticos, históricos, etnológicos, naturales y científico-tecnológicos, dando sentido e identificando a una determinada sociedad (Estepa-Giménez, 2013).

Esta visión holística del patrimonio está muy relacionada con la necesaria interdisciplinariedad de las didácticas de las ciencias, que desde hace bastante tiempo se viene discutiendo por autores 
como Porlán (1990), más proclive a una visión más auténtica al hablar de alfabetización científica cultural en las didácticas de las ciencias experimentales y sociales.

\subsection{Las emociones y la identidad patrimonial}

Desde el punto de vista de la didáctica de las ciencias experimentales se abre un nuevo cauce a la C-T-S y al enfoque de enseñanza contextualizada, reforzando la función emocional con las señas de identidades locales. Esto es la consecuencia de esa evolución de la concepción patrimonial (Estepa Giménez, Wamba Aguado y Jiménez Pérez, 2005), en cuanto al papel que exclusivamente jugaba el patrimonio en la educación, desde la mera ilustración en la geografía y la historia hasta el patrimonio como recurso, para el conocimiento del entorno social, cultural y natural.

Algunos autores (Moraga, Espinet y Merino, 2019) proponen que una enseñanza contextualizada es más productiva y motivadora, capaz de reconocer la utilidad del conocimiento que se aprende, estimulando la necesidad de aprender, generando emociones positivas al descubrir retos que llevan a formular preguntas estimulantes con elementos patrimoniales identitarios permitiendo conocer el entorno, la historia de la ciencia y la tecnología que junto a habilidades de investigación, comunicación, búsqueda de información y pensamiento creativo, son las bases de una fructífera confrontación de ideas y toma de decisiones ante problemas reales y socialmente útiles.

La educación se está haciendo eco de esta necesidad introduciéndose poco a poco en los curricula a través de metodologías más abiertas y flexibles, utilizando contextos adecuados (Caamaño, 2011) y emocionalmente positivos (Mellado et al., 2014) con el legado tecnológico como recurso, que además de ser elemento identitario del entorno cercano (Hernández y Guillén, 2017), ha tenido consecuencias sociales formando parte de la historia social, medioambiental, etnológica, arqueológico y natural.

Por otra parte, como señala Mattozzi (2001) un bien cultural forma parte de un sistema y su comprensión se incrementa con relaciones de sincronía, diacronía, génesis, analogía o diferencia. La relación intrínseca entre lo social y lo natural o socionatural, en entornos transformados, es un determinante del sistema patrimonial. La obtención de recursos para actividades económicas, resultados de procesos de interacción, pueden llegar a adquirir un valor o una consideración que les distinga del resto que, consciente o inconscientemente, pueden determinar un hecho patrimonial, por criterios de antigüedad, estéticos o de carácter identitario (Cuenca, 2002), con mayor posibilidad de aumentar las emociones positivas.

Por otro lado, el vínculo existente entre las emociones y la identidad patrimonial es, en muchas ocasiones, involuntario, provocando cambios de carácter intrapersonales e interpersonales. Estas reacciones, a priori involuntarias, son las que nos sirven de base para el procesamiento emocional de la información (Cuenca-López y Estepa-Giménez, 2017). De ahí la importancia, en este trabajo, en analizar los cambios de emociones que se producen el alumnado con el conocimiento de un elemento patrimonial tecnológico identitario y su utilidad para explicar un proceso químico (Retana-Alvarado, de las Heras Pérez, Vázquez-Bernal y Jiménez-Pérez, 2018).

Para concluir este apartado, deseamos poner el foco de atención en la importancia del sexo del alumnado respecto a las emociones en estos contextos de aprendizajes. Algunos estudios desarrollados con alumnado de ESO apuntan a la existencia de diferencias significativas, en lo que respecta a las competencias emocionales de varones y mujeres en la capacidad para comprender emociones complejas y, a veces, contradictorias en su manejo (Peñalva-Vélez, López-Goñi y GarcíaManso, 2016; Andrade, Cruz, Guerrero y Rodríguez, 2014). Otros estudios, sin embargo, con alumnado mayor de edad, no aprecian diferencias relevantes y significativas, dado que la capacidad para el manejo y funcionamiento de las habilidades emocionales están inherentes, tanto para los alumnos como para las alumnas indistintamente y sin diferenciación alguna (Álvarez, 2015). Se puede deducir, por tanto, que no existe una tendencia general al respecto, sino que es muy dependiente 
del contexto, por lo que parece interesante estudiarlo en este trabajo y extraer consecuencias posibles relevantes.

\section{METODOLOGÍA DE LA INVESTIGACIÓN}

\subsection{Preguntas de investigación}

El problema principal de esta investigación es ¿Cómo son las emociones que experimentan alumnos/as de $2^{\circ}$ de ESO cuando se trabaja un recurso patrimonial del entorno en un contexto de aprendizaje de la química? De este problema principal se derivan los siguientes problemas:

- ¿Cómo cambian las intensidades de las emociones antes, durante y después de la experiencia y cuál es la naturaleza de los cambios?

- ¿Existen variaciones respecto al sexo en estas emociones?

- ¿Cuáles son las posibilidades de uso de un elemento patrimonial como recurso en las clases de Ciencias Experimentales?

- ¿Está justificado su inclusión en las actividades habituales de la materia a pesar de su coste?

\subsection{Contexto y sujetos}

La investigación que se presenta se enmarca dentro de un paradigma interpretativo, utilizando una metodología mixta, donde se espera que el investigador recoja, analice los datos rigurosamente en respuesta a las preguntas de investigación, combine sus resultados, los organice en diseños específicos y los encuadre dentro de un contexto teórico (Creswell y Plano, 2018). El trabajo se desarrolló en un grupo natural de $2^{\circ}$ de ESO que cursaba la asignatura de Física y Química, en concreto la unidad didáctica "La Materia" (los objetivos, criterios de evaluación, competencias y estándares implicados se encuentran en el anexo I). Para trabajar los métodos de separación de las mezclas se acordó con el grupo de investigación EPITEC (Educación Patrimonial para la Inteligencia Territorial y Emocional) realizar una visita a un elemento patrimonial de la zona, Molino de mareas, para observar su funcionamiento y vincularlo al estudio de ese contenido (la visita cuesta entre 6 y $12 €)$. La idea fundamental era que todo estuviera inscrito dentro del propio proceso enseñanzaaprendizaje de la asignatura, asociándolo a un tópico concreto y a un desarrollo basado en las buenas prácticas docentes.

La muestra empleada fue natural, de naturaleza incidental por razones de accesibilidad y conveniencia (muestreo no probabilístico). Estuvo conformada por 17 alumnos/as procedentes de un centro de Enseñanza Secundaria de la Bahía de Cádiz (España), de los cuales 11 eran varones (65\%) y 6 mujeres (35\%), oscilando entre edades de 13 y 15 años, con una edad media de 13,41 años (DE $=0,80)$. El alumnado del centro pertenece a un nivel socioeconómico medio-bajo según los términos ofíciales de la Junta de Andalucía.

\subsection{Descripción de la Intervención}

La visita estaba enmarcada dentro de un proceso de Indagación Escolar orientada al aprendizaje de los aspectos relacionados con los métodos de separación de las mezclas, con la idea de que el diseño instruccional influyera positivamente en el aprendizaje del alumnado (Hernández, Couso y Pintó, 2015). Se consideraron un conjunto de fases (Estepa, 2007), sustentadas por un repertorio de actividades. En la fase de iniciación, planificación y búsqueda de información, el alumnado realizó diferentes actividades (Anexo I, actividades 1 a 6), con la extracción de sólidos (en concreto la harina) como núcleo vertebrador, para tratar de relacionarlo con la molturación del grano en el Molino de Mareas. La planificación incluía una delimitación más precisa del problema planteado en el aula “Qué uso tenía el molino de Mareas y cómo se desarrollaba?” (Molino Zaporito, en anexo II). Este 
proceso incluyó la búsqueda de información, donde el alumnado, con la ayuda de Internet y desde una perspectiva histórica, recopiló y resumió el uso de la molturación del trigo desde la antigüedad y su importancia para las sociedades humanas. Al final de esta fase, el alumnado rellenó el primer cuestionario sobre las emociones previas a la visita.

En el momento de la visita, se volvió a aplicar el cuestionario, justo cuando esta concluyó; posteriormente, en la fase de estructuración y evaluación, ya de vuelta en el centro, se realizó una reflexión grupal e individual y un conjunto de nuevas actividades (actividades 7 a 13 del Anexo I), que incluyeron la proyección de imágenes de un Molino de mareas en estado ruinoso (Río Arillo; anexo II). Transcurridos aproximadamente una semana, se volvió a completar el cuestionario de emociones.

\subsection{Instrumentos de la Investigación}

Desde una perspectiva metodológica mixta, se ha tratado de complementar dos visiones, una nomotética, que busca el acercamiento a grupos humanos a través de la búsqueda de tendencias estadísticas y otra, ideográfica, que pone el centro de interés en el individuo (Colás y Buendía, 1998), en nuestro caso lo afectivo e identitario.

El trabajo indaga en dos conceptos claves: Emociones e Identidad Patrimonial. Respeto al primero, existen tres grandes tradiciones de las emociones desde una perspectiva histórica: sentimental, motivacional y evaluativa (Scarantino, 2018). Nuestra postura teórica está más cerca de la primera tradición, para quien las emociones son fenómenos que emergen en la conciencia en el momento a partir de ingredientes más fundamentales o afectos nucleares (excitación, tranquilidad, agrado y desagrado). En este sentido, lo que llamamos emociones son, en realidad, categorías emocionales, porque cada una es un conjunto de casos diversos (Barrett, 2017). Por su parte, la identidad patrimonial, como concepto dinámico en el tiempo, son los sentimientos que experimentan los miembros de una colectividad que se reconocen en las expresiones que han configurado su cultura (Hart Dávalos, 2003; Piedra Sarría, 2011).

Para dar respuesta a las dos primeras preguntas de investigación de naturaleza cuantitativa (cambio de emociones e influencia del sexo), el instrumento de recogida de información para conocer el cambio de las emociones respecto a la salida fue un cuestionario-catálogo o Taxonomía de emociones ampliamente utilizado (Borrachero, Brígido, Gómez, Bermejo y Mellado, 2011; Borrachero, Brígido, Mellado, Costillo y Mellado, 2014) que consta de 22 variables-emociones en una escala tipo Likert de cinco opciones de elección: 1 (totalmente en desacuerdo), 2 (desacuerdo), 3 (de acuerdo), 4 (bastante de acuerdo) y 5 (totalmente de acuerdo). En este sentido, la razón de una escala de cinco es que se ha confirmado que la confiablidad aumenta (Matas, 2018). En la Tabla 1, se exponen las emociones recogidas en el cuestionario.

Para comprobar si la muestra provenía de poblaciones con distribución normal se usó, en los tres momentos, la prueba inferencial no paramétrica de Kolmogorov-Smirnov. Los resultados fueron dispares, así, en 32 variables se aceptó la hipótesis nula con significancias menores que el nivel de significación estadística del 5,00\% (p-valor $<0,05$ ); en cambio, en 29 variables, se rechazaron las hipótesis nulas (H0) y se aceptaron las hipótesis alternativas (H1), es decir, provenían de distribuciones no normales; además, en 5 ocasiones no se pudo realizar el cálculo. Por tanto, para la realización de pruebas, dada la diversidad de distribuciones (normales 48,5\%; no normales $44 \%$ y no calculadas 7,5 \%), se decidió aplicar la prueba no paramétrica de Kruskal Wallis.

En base a una investigación mixta, el segundo instrumento de recogida de información fueron las producciones del alumnado obtenidas en las fases del proyecto de aula (actividades 1 a 13 del Anexo I). En este conjunto de actividades aparecen, además de cuestiones conceptuales y procedimentales no analizadas en este artículo, otras que hacen alusión a la parte afectiva y de pertenencia a un entorno (actividades 8 y 13). Se analizaron en base a dar respuesta al tercer problema planteado (posibilidades de uso del Molino) y, de ahí, obtener las unidades de información más 
relevantes en este sentido que, como acabamos de expresar, implicaran respuestas que entraran dentro de las categorías Emociones e Identidad Patrimonial. Por su parte, el problema cuarto (toma de decisiones), se abordaría por la triangulación de los resultados obtenidos en los problemas anteriores.

TABLA 1. Taxonomía de las emociones

\begin{tabular}{cc}
\hline Emociones positivas & Emociones negativas \\
\hline Alegría & Aburrimiento \\
Confianza & Ansiedad \\
Diversión & Depresión \\
Entusiasmo & Desesperación \\
Motivación & Frustración \\
Gratificación & Miedo \\
Orgullo & Nerviosismo \\
Placer & Odio \\
Satisfacción & Pesimismo \\
Simpatía & Preocupación \\
Tranquilidad & Tensión \\
\hline
\end{tabular}

Fuente: Borrachero et al., 2011

\section{RESULTADOS DE LA INVESTIGACIÓN}

\subsection{Análisis de fiabilidad y descriptive del cuestionario de emociones}

La fiabilidad interna del cuestionario-catálogo de emociones, en términos de alfa de Cronbach, mostraron valores de 0,89 para todo el cuestionario completo y valores de 0,78 para el de antes de la visita; 0,80 durante y 0,79 para después de la visita, cifras satisfactorias en una investigación exploratoria (Robinson, Shaver y Wrightsman, 1991).

En la Tabla 2 se exponen los resultados de las emociones y sus intensidades en los tres momentos. Antes de la intervención, las emociones más intensas en orden descendiente fueron: Diversión (4,18), Alegría (3,76), Tranquilidad (3,59), Entusiasmo (3,59), Simpatía (3,47), Confianza $(3,35)$, Gratificación $(2,94)$, Motivación $(2,94)$, Satisfacción $(2,88)$, Orgullo $(2,65)$ y Placer $(2,53)$. Con respecto a las emociones negativas, de mayor intensidad a menor, el orden fue: Aburrimiento $(2,06)$, Nerviosismo (1,41), Depresión $(1,24)$, Miedo $(1,18)$, Frustración $(1,12)$, Pesimismo $(1,12)$, Preocupación $(1,12)$, Desesperación $(1,06)$, Tensión $(1,06)$, Ansiedad $(1,00)$ y Odio $(1,00)$. Llama la atención que entre las más intensas se encuentren pares de emociones opuestas Diversión/Aburrimiento, en la misma medida que las menos intensas Placer/Odio. Se observa, pues, una gran diferencia entre las emociones positivas y negativas, destacando Diversión del alumnado con cierta dosis de posible Aburrimiento, como síntesis.

Durante la visita, por su parte, en orden decreciente de intensidad de emociones positivas fueron: Diversión (4,35), Alegría $(4,18)$, Confianza (3,94), Tranquilidad (3,94), Entusiasmo $(3,71)$, Motivación (3,71), Simpatía (3,59), Gratificación $(3,47)$, Orgullo $(3,47)$, Satisfacción $(3,35)$ y Placer $(2,76)$. Por su parte, las emociones negativas hallamos: Aburrimiento $(1,76)$, Frustración $(1,35)$, Desesperación (1,18), Miedo (1,18), Nerviosismo (1,18), Ansiedad (1,12), Depresión $(1,12)$, Tensión $(1,00)$, Odio $(1,00)$, Pesimismo $(1,00)$ y Preocupación $(1,00)$. Vuelven a repetirse los pares más y menos intensos. En síntesis, la "foto" sintética de las emociones es algo más positivo, la Diversión que acontece en ese momento y disminuye el Aburrimiento real.

Después de la salida, las emociones más intensas son ahora: Diversión $(3,76)$, Alegría $(3,59)$, Tranquilidad (3,59), Confianza (3,53), Gratificación (3,53), Orgullo (1,89), Entusiasmo $(3,41)$, 
Motivación $(3,41)$, Simpatía $(3,41)$, Satisfacción $(3,35)$ y Placer $(2,71)$; con respecto a las negativas: Aburrimiento (2,06), Frustración (1,47), Odio (1,35), Pesimismo (1,35), Depresión $(1,29)$, Desesperación (1,29), Ansiedad (1,18), Preocupación $(1,18)$, Tensión $(1,12)$, Miedo $(1,06) \mathrm{y}$ Nerviosismo $(1,06)$. El par más intenso vuelve a ser Diversión/Aburrimiento y ahora el menos intenso Placer/Nerviosismo-Miedo. En general, disminuyen la intensidad de las emociones, excepto alguna subida interesante como es el Odio, que volveremos sobre él.

TABLA 2. Estadísticos (M media, DE desviación estándar, Mdn mediana y S2 varianza) sobre las emociones y sus intensidades en los tres momentos de la visita

\begin{tabular}{ccccc|cccc|cccc}
\hline & \multicolumn{4}{c|}{ Antes } & \multicolumn{4}{c|}{ Durante } & \multicolumn{4}{c}{ Después } \\
\hline Alegría & $\mathbf{M}$ & $\mathbf{D E}$ & $\mathbf{M d n}$ & $\mathbf{S}^{\mathbf{2}}$ & $\mathbf{M}$ & $\mathbf{D E}$ & $\mathbf{M d n}$ & $\mathbf{S}^{\mathbf{2}}$ & $\mathbf{M}$ & $\mathbf{D E}$ & $\mathbf{M d n}$ & $\mathbf{S}^{\mathbf{2}}$ \\
Confianza & 1,145 & 4,00 & 1,31 & 4,18 & 1,24 & 5,00 & 1,53 & 3,59 & 1,54 & 4,00 & 2,38 \\
Diversión & 4,18 & 1,32 & 3,00 & 1,74 & 3,94 & 1,35 & 5,00 & 1,81 & 3,53 & 1,42 & 4,00 & 2,012 \\
Entusiasmo & 3,59 & 1,46 & 4,00 &, 90 & 4,35 & 1,06 & 5,00 & 1,12 & 3,76 & 1,56 & 5,00 & 2,44 \\
Motivación & 2,94 & 1,44 & 4,00 & 2,06 & 3,71 & 1,26 & 4,00 & 1,60 & 3,41 & 1,58 & 4,00 & 2,51 \\
Gratificación & 2,94 & 1,35 & 3,00 & 1,81 & 3,47 & 1,50 & 4,00 & 1,97 & 3,41 & 1,58 & 3,00 & 2,51 \\
Orgullo & 2,65 & 1,37 & 2,00 & 1,87 & 3,47 & 1,38 & 3,00 & 1,89 & 3,53 & 1,42 & 4,00 & 2,02 \\
Placer & 2,53 & 1,28 & 3,00 & 1,64 & 2,76 & 1,60 & 3,00 & 2,57 & 2,71 & 1,65 & 2,00 & 2,72 \\
Satisfacción & 2,88 & 1,41 & 3,00 & 1,96 & 3,35 & 1,62 & 4,00 & 2,62 & 3,35 & 1,73 & 3,00 & 2,99 \\
Simpatía & 3,47 & 1,18 & 3,00 & 1,39 & 3,59 & 1,37 & 4,00 & 1,88 & 3,41 & 1,54 & 4,00 & 2,38 \\
Tranquilidad & 3,59 & 1,23 & 4,00 & 1,51 & 3,94 & 1,92 & 4,00 & 1,43 & 3,59 & 1,54 & 4,00 & 2,38 \\
Aburrimiento & 2,06 & 1,25 & 2,00 & 1,56 & 1,76 & 1,30 & 1,00 & 1,69 & 2,06 & 1,48 & 1,00 & 2,18 \\
Ansiedad & 1,00 & 0,00 & 1,00 & 0,00 & 1,12 & 0,33 & 1,00 & 0,11 & 1,18 & 0,39 & 1,00 & 0,15 \\
Depresión & 1,24 & 0,97 & 1,00 & 0,94 & 1,12 & 0,33 & 1,00 & 0,11 & 1,29 & 0,69 & 1,00 & 0,47 \\
Desesperación & 1,06 & 0,24 & 1,00 & 0,06 & 1,18 & 0,53 & 1,00 &, 28 & 1,29 & 0,69 & 1,00 & 0,47 \\
Frustración & 1,12 & 0,33 & 1,00 & 0,11 & 1,35 & 1,06 & 1,00 & 1,12 & 1,47 & 1,07 & 1,00 & 1,14 \\
Miedo & 1,18 & 0,53 & 1,00 & 0,28 & 1,18 & 0,73 & 1,00 & 0,53 & 1,06 &, 24 & 1,00 & 0,06 \\
Nerviosismo & 1,41 & 0,87 & 1,00 & 0,76 & 1,18 & 0,73 & 1,00 & 0,53 & 1,06 &, 24 & 1,00 & 0,06 \\
Odio & 1,00 & 0,00 & 1,00 & 0,00 & 1,00 & 0,00 & 1,00 & 0,00 & 1,35 & 1,00 & 1,00 & 0,99 \\
Pesimismo & 1,12 & 0,33 & 1,00 & 0,11 & 1,00 & 0,00 & 1,00 & 0,00 & 1,35 & 1,00 & 1,00 & 0,99 \\
Preocupación & 1,12 & 0,33 & 1,00 & 0,11 & 1,00 & 0,00 & 1,00 & 0,00 & 1,18 &, 53 & 1,00 & 0,28 \\
Tensión & 1,06 & 0,24 & 1,00 & 0,59 & 1,12 & 0,49 & 1,00 & 0,24 & 1,12 &, 33 & 1,00 & 0,11 \\
\hline & & & & & & & & & & & &
\end{tabular}

Fuente: Elaboración propia

Las diferencias existentes en los tres momentos de la salida pueden observarse en la Figura 1, donde las emociones "durante" son ligeramente más intensas, tendencia que se da en las emociones negativas en el "después". 
FIGURA 1. Valores medios del conjunto de las emociones en los tres momentos de la visita al molino

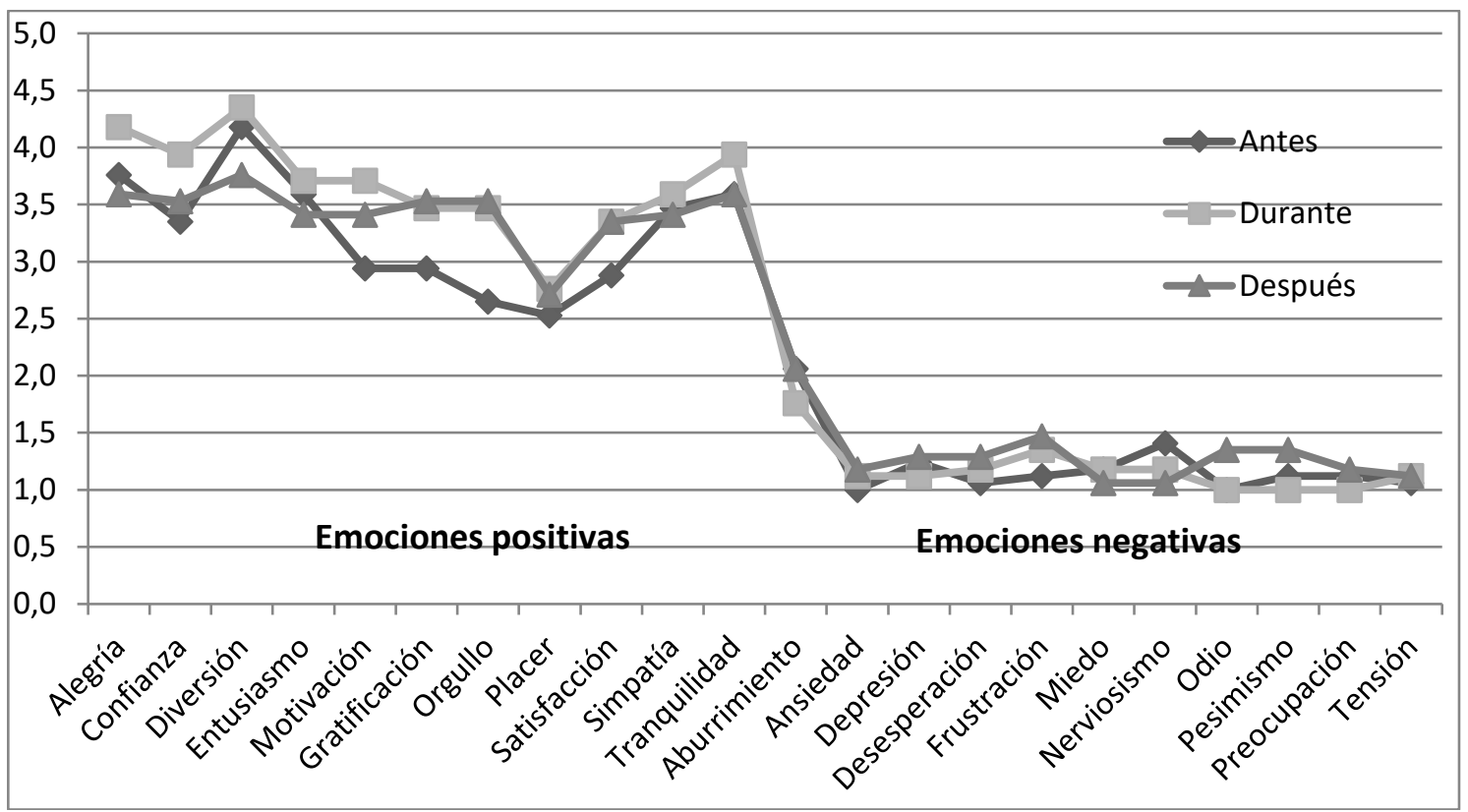

Fuente: Elaboración propia

\subsection{Pruebas de hipótesis}

En la Tabla 3, se muestran los resultados tras aplicar la prueba no paramétrica $H$ de KruskalWallis para tres muestras independientes $(\mathrm{N}=17)$, en función de los tres momentos investigados, en la búsqueda de diferencias significativas para cada variable. La hipótesis nula establece que existen tales diferencias. Se observa que solo la variable Odio muestra diferencias significativas en los tres momentos de la visita $(\mathrm{p}=0,044)$. Si acudimos a las Tabla 2, veremos que es el Después de la visita la fase que arroja un valor medio superior $(1,35)$, respecto a los momentos precedentes $(1,00)$, aunque el error relativo de la medida es del $74 \%$, indicándonos que esos cambios afectan a un número de alumnos/as muy limitados. El cálculo de la potencia estadística (42\%, un valor medio-bajo) y el valor del coeficiente eta cuadrado $(E 2 R=0,39)$, confirman que, aunque limitado, el tamaño del efecto es destacable.

Se aplicó la misma prueba no paramétrica H de Kruskal-Wallis para ver si existían diferencias significativas respecto al sexo en todas las emociones y en los tres momentos, hallándose que no existía ninguna diferencia significativa. Es un caso interesante, ya que en varios estudios (Dávila Acedo, Borrachero Cortés, Mellado Jiménez y Bermejo García, 2015; Chittum y Jones, 2017; Khishfe, Alshaya, BouJaoude, Mansour y Alrudiyan, 2017; Strati, Schmidt y Maier, 2017), la variable sexo suele jugar un papel discriminante, pero no es nuestro caso. 
TABLA 3. Prueba no paramétrica $H$ de Kruskal-Wallis para tres muestras independientes. *p $<0,05$

\begin{tabular}{ccccc}
\hline Emociones & $\mathbf{X}^{\mathbf{2}}$ & $\mathbf{p}$ & Potencia & $\mathbf{E}^{2} \mathbf{R}$ \\
\hline Alegría & $2,144($ retiene Ho) & 0,342 & - & - \\
Confianza & 1,831 & 0,400 & - & - \\
Diversión & 1,233 & 0,540 & - & - \\
Entusiasmo &, 169 & 0,919 & - & - \\
Motivación & 2,422 & 0,298 & - & - \\
Gratificación & 2,042 & 0,360 & - & - \\
Orgullo & 4,165 & 0,125 & - & - \\
Placer &, 148 & 0,929 & - & - \\
Satisfacción & 1,109 & 0,574 & - & - \\
Simpatía &, 186 & 0,911 & - & - \\
Tranquilidad &, 886 & 0,642 & - & - \\
Aburrimiento & 1,444 & 0,486 & - & - \\
Ansiedad & 3,043 & 0,218 & - & - \\
Depresión & 1,033 & 0,597 & - & - \\
Desesperación & 1,241 & 0,538 & - & - \\
Frustración & 1,175 & 0,556 & - & - \\
Miedo & 0,504 & 0,777 & - & - \\
Nerviosismo & 3,259 & 0,196 & - & - \\
Odio & 6,245 & $0,044 *$ & $42 \%$ & 0,39 (efecto fuerte: $\left.0,04<\mathrm{E}^{2}{ }_{\mathrm{R}} \leq 0,36\right)$ \\
Pesimismo & 3,091 & 0,213 & - & - \\
Preocupación & 2,127 & 0,345 & - & - \\
Tensión & 0,473 & 0,789 & - & - \\
\hline
\end{tabular}

Fuente: Elaboración propia

\subsection{Uso de un recurso patrimonial en las clases de Ciencias Experimentales: emoción e identidad patrimonial}

Para la aproximación cualitativa, se han seleccionado 5 alumnos/as dentro los participantes, principalmente porque el resto se expresaba en términos muy similares o sus respuestas eran muy escuetas o breves. En cuanto a los resultados obtenidos sobre el uso del recurso patrimonial y atendiendo a las respuestas del alumnado en sus producciones (actividades 8 y 13), se observa en un grupo reducido de alumnas esa explicitación de la desilusión que, según interpretamos, puede haber ayudado al leve aumento, aunque significativo, del Odio en el momento posterior a la visita, ya que la emoción más cercana a su sentimiento de desilusión es la Frustración en el cuestionario, una emoción que al alumnado le cuesta reconocer y suele establecer complejas asociaciones (Earl, Taylor, Meijen y Passfield, 2017): "Desilusión al estar cambiado del todo. Creo que sería mejor dejarlo como antes..." (Alumna 1, respuesta actividad 8); "Desilusión al saber que lo han modernizado completamente por dentro y saber que no lo han dejado como antiguamente... (Alumna 1, respuesta actividad 13); "Un poco de desilusión. Lo han cambiado completamente por dentro y sería dejarlo como estaba... (Alumna 2, respuesta actividad 8).

También, esta diferencia puede tener relación con las imágenes mostradas al alumnado del molino en estado ruinoso: "Está muy modernizado por dentro y no como antiguamente... (Alumna 1 , respuesta actividad 13).

Por otro lado, la valoración y el vínculo generado con el territorio (Identidad Patrimonial), a tenor de sus producciones, son bastante positivos, más allá de los aspectos lúdicos: "Me ha parecido una buena experiencia saber cómo era San Fernando antes y cómo se llamaba El Molino y que lo 
inspiró, cómo fue su vida, que al final lo hicieron en honor a Zaporito y la gran importancia que tuvo el molino" (Alumno 3, actividad 8).

"Me lo pasé muy bien, me encantó la historia de cómo poco a poco se fue creando San Fernando, también me gustó mucho la historia de la familia que creó el caño artificial y los barcos que llegaban para comerciar" (Alumno 4, actividad 8).

También se denota cómo diversos alumnos/as se sienten satisfechos por conocer la historia de su ciudad, sus personajes y los lugares históricos que lo constituyen, a partir de la visita a un elemento patrimonial del entorno próximo: "Estar allí me ha hecho pensar imaginarme la historia de San Fernando" (Alumno 5, actividad 8).

"Cuando entré allí me quedé asombrada, la mujer que trabajaba allí nos contó la historia del molino y quiénes lo hicieron. El apellido de uno de los hombres que pasó por allí fue Zaporito fue todo muy divertido" (Alumno 16, actividad 8).

"Me divertí mucho y me encantó la historia del molino, me he quedado con ganas de saber más, fue muy curioso y divertido" (Alumno 4, actividad 8).

\section{CONCLUSIONES}

En respuesta al primer problema planteado, los cambios en las emociones han mostrado una evolución interesante, con tendencias levemente superiores in situ a las emociones previas a la visita y con descensos posteriores, aunque intermedio, en general, con el paso del tiempo y su recuerdo. La experiencia puede denominarse como constructiva, en cuanto a las emociones positivas mostradas en los diferentes momentos, siempre sensiblemente más elevadas que las negativas. Además, se ha visto que las emociones deparan sorpresas en las respuestas del alumnado, pero lejos de ser un inconveniente, nos da la posibilidad de reflexionar sobre su naturaleza y nos ha permitido pergeñar que la restauración de los elementos patrimoniales, su uso y disfrute del ciudadano, a veces, confronta con afectos inesperados.

Incluir elementos afectivos en el aula de ciencias experimentales tiene como finalidad adquirir competencias que favorezcan el bienestar personal y social, un constructo al que solemos denominar como inteligencia emocional (Bisquerra, 2016), muy emergente hoy en día, que ejerce una influencia mediadora entre el clima de aula y el rendimiento académico (López-González y Oriol, 2016). El papel de las emociones ante elementos patrimoniales está descrito en la bibliografía. Así, Santacana (2007) aduce que, en un mismo observador, puede suscitar la confluencia de varias emociones cruzadas, positivas y negativas (Amor y Odio). En nuestro trabajo, se ha observado cómo, en efecto, el elemento analizado, Molino del Zaporito, puede provocar cambios en emociones inesperados para los investigadores, por sentimientos encontrados entre el alumnado. Nos referimos al odio y su diferencia significativa entre los tres momentos analizados.

En lo que respecta a la influencia del sexo, en nuestra pequeña muestra no se ha mostrado cambio de las emociones, ni en las intensidades. Ahora bien, es revelador que fuesen mujeres las que especialmente sintieran y supieran explicar el efecto de ver el Molino del Zaporito dedicado a otras actividades y contribuyeran a subir la intensidad de la emoción Odio después de la visita. Esto es compatible con algunos estudios que muestran que las mujeres revelan más estrés que los varones en experiencias innovadoras (Schneider et al., 2016).

Centrándonos en el tercer problema, prensamos que el uso ha despertado una motivación en el alumnado hacia su entorno, más allá de las operaciones tradicionales que suelen usarse en los métodos de separación de sustancias, como atestiguan sus expresiones al ser preguntados. La inclusión de elementos patrimoniales en el aprendizaje de las ciencias experimentales vinculado a las ciencias sociales, afianza una visión integradora y cercana de las prácticas en el aula y garantiza, aprovechando la dimensión afectiva del patrimonio, el conocimiento, preservación y transmisión del mismo en todas sus dimensiones (Etxeberria, Merillas y López, 2015), además reporta beneficios pedagógicos en el 
desarrollo de la conciencia histórica del alumnado (Chaparro Sainz, Martínez Hernández, Robles Moral y Cepedonsa Rojas, 2019).

Por su parte, en nuestro caso, como hemos indicado, nos permite relacionarlo con procesos físico-químicos esenciales del currículo (separación de sustancias y sus procedimientos,...) que, a veces parecen alejados de la sociedad, pero que hunden sus raíces en los procesos de desarrollo histórico, lo cual supone un cambio de paradigma en la enseñanza científica, de manera que se deje atrás la finalidad propedéutica de las materias de ciencias en la ESO y la educación se oriente hacia una Alfabetización Científica (Merchán-Galán, 2018), máxime cuando en los propios currículos actuales su presencia es insuficiente (Fernandes, Pires y Delgado-Iglesias, 2018) o, en el peor de los casos, se trate de forma poco crítica y superficial, sin considerar los propósitos del desarrollo científico-tecnológico y sus consecuencias sociales (Strieder, Torija y Quílez, 2017).

En el análisis del contenido de las producciones del alumnado se destaca ese sentimiento de pertenencia a un territorio, de lo que se ha denominado Inteligencia Territorial, esto es, la construcción de identidades locales que se construyen, en gran medida, gracias a la difusión del patrimonio (Lacour, 2008). El alumnado muestra asombro, agrado y ganas de aprender más de la historia de su localidad. Nos parece muy positivo la idea de un espacio surcado de información y cultura, comunicación y participación, enriquecido con la reflexión y la emotividad (Rovetta, Rovida y Zafferri, 2017) que proyectos como el implementado pueden generar en el alumnado.

El abordaje del patrimonio y su conservación desde las ciencias experimentales es, en especial desde la Física-Química, interesante, pues vincula el entorno local del alumnado a una visión holística, fomentando procesos dirigidos a la posible readquisición de las competencias para proteger naturaleza, condiciones de vida y patrimonio histórico-natural-artístico (Lorusso, Cogo y Natali, 2017). Una visión que contrasta con lo encontrado en España, donde analizados un amplio catálogo de libros de editoriales afianzadas, los elementos históricos y artísticos destacan sobre otras manifestaciones del patrimonio (Cuenca y López-Cruz, 2014).

De la triangulación de los resultados obtenidos (cuarto problema), tanto desde una perspectiva grupal como individual, pensamos, sinceramente, que la inclusión del Molino y las actividades que se programaron están justificadas. La experiencia y su efecto nos ha ayudado a la toma de decisiones futuras sobre la participación del alumnado en nuevos ciclos, tomando el molino del Zaporito como elemento patrimonial, para consolidar lo que Bertacchini (2016) denomina Inteligencia Territorial, donde los actores locales, en nuestro caso el alumnado, se apropian de los recursos de un espacio, movilizando y transformando la energía del sistema territorial en capacidad de proyecto. Fue interesante abordar los procedimientos de separación de sustancias a través de una metodología investigadora con el protagonismo del alumnado (Rite y López, 2017), incidiendo, en factores patrimoniales y afectivos que ayuden a investigar sobre el desarrollo de la localidad.

Una de las evidentes limitaciones del estudio es el pequeño tamaño de la muestra, sin embargo, está en consonancia con uno de los problemas del estudio, ayudar a la toma de decisiones sobre el uso futuro del recurso patrimonial. Debido a que los resultados desde el punto de vista afectivo fueron muy positivos, con un cambio significativo y de efecto tamaño fuerte en una sola emoción (Odio: algo imprevisto ya analizado en el trabajo), creemos que aportan una base sólida para nuevas salidas al Molino, ya que su uso supone un desembolse extra de dinero para las familias y, por tanto, debe estar justificado en una zona con economía no pujante.

En la actualidad estamos evaluando los aprendizajes del alumnado desde el punto de vista físico-químico como complemento a la parte emocional y para rediseñar, en caso necesario, las actividades. 


\section{Referencias}

Acevedo Díaz, J.A., García-Carmona, A. y Aragón, M.M. (2016). Un caso de Historia de la Ciencia para aprender Naturaleza de la Ciencia: Semmelweis y la fiebre puerperal. Revista Eureka sobre Enseñanza y Divulgación de las Ciencias, 13(2), 408-422.

Álvarez, R.M.B. (2015). Inteligencia emocional y rendimiento académico en alumnos del 2 Año de la Escuela de Educación Secundaria. Revista de Psicología, 7, 78-96.

Andrade, N.A.O., Cruz, R.G., Guerrero, A.M.R. y Rodríguez, D.J. (2014). Las emociones de jóvenes universitarios con respecto al sexo, rendimiento escolar y trayectoria escolar. Un estudio comparativo. European Scientific Journal, ESJ, 10(10), 219-227. DOI: 10.19044/esj.2014.v10n10p/25p

Barrett, L.F. (2017). How Emotions are Made: The Secret Life of the Brain. Houghton Mifflin Harcourt.

Bertacchini, Y. (2016). Intelligence territoriale. intelligence, Entre Savoirs y Expérimentation (1). Collection Les ETIC. Toulon (France): Presses Technologiques.

Bisquerra, R. (2016). Educación emocional. Documento inédito elaborado para las Jornadas del Máster en Resolución de Conflictos en el Aula.

Borrachero, A.B., Brígido, M., Gómez, R., Bermejo, M.L. y Mellado, V. (2011). Las emociones de los futuros profesores de secundaria sobre el aprendizaje y la enseñanza de las ciencias. International Journal of Developmental and Educational Psychology. INFAD Revista de Psicología, 3(1), 521-530.

Borrachero, A.B., Brígido, M., Mellado, L., Costillo, E. y Mellado, V. (2014). Emotions in prospective secondary teachers when teaching science content, distinguishing by gender. Research in Science y Technological Education, 32(2), 182-215. DOI: 10.1080/02635143.2014.909800

Caamaño, A. (2011). Enseñar química mediante la contextualización, la indagación y la mdelización. Alambique, 69, 21-34

Castro-Martínez, E. (2018). Los procesos de producción, intercambio y transferencia de conocimiento en las ciencias humanas y sociales: especificidades del patrimonio cultural. Revista PH, 95, 88-101.

CEJA (Consejería Educación Junta de Andalucía) (2016a). Decreto 111/2016, de 14 de junio, por el que se establece la ordenación y el currículo de la educación Secundaria obligatoria en la comunidad Autónoma de Andalucía. BOJA, 122, 27-45.

CEJA (Consejería Educación Junta de Andalucía) (2016b). Orden de 14 de julio de 2016, por la que se desarrolla el currículo correspondiente a la educación Secundaria Obligatoria en la Comunidad Autónoma de Andalucía. BOJA, 114, 108-396.

Chaparro Sainz, A., Martínez Hernández, C., Robles Moral, F.J. y Cepedonsa Rojas, R. (2019). Desarrollar la conciencia histórica a través del patrimonio en el Grado en Educación Primaria. Didáctica de las ciencias experimentales y sociales, 36, 17-32. DOI: 10.7203/DCES.36.12672

Chittum, J.R., y Jones, B.D. (2017). Identifying pre-high school students' science class motivation profiles to increase their science identification and persistence. Journal of Educational Psychology, 109(8), 1163-1187. DOI: 10.1037/edu0000176

Creswell, J.W. y Plano, V.L. (2018). Designing and Conducting Mixed Methods Research. Third Edition. London: Sage Publications.

Colás, M.P. y Buendía, L. (1998). Investigación Educativa. Sevilla: Alfar.

Cuenca, J.M. (2002). El patrimonio en la Didáctica de las ciencias sociales. Análisis de concepciones, dificultades y obstáculos para su integración en la enseñanza obligatoria. Tesis doctoral. Universidad de Huelva. 
Cuenca-López, J.M. y Estepa-Giménez, J. (2017). Educación patrimonial para la inteligencia territorial y emocional de la ciudadanía. MIDAS, 8. Recuperado de http://journals.openedition.org/midas/1173. DOI: 10.4000/ midas.1173

Cuenca, J.M., Estepa-Giménez, J. y Martín-Cáceres, M.J. (2017). Patrimonio, educación, identidad y ciudadanía. Profesorado y libros de texto en la enseñanza obligatoria. Revista de Educación, 375, 136-159. DOI: 10.4438/1988-592X-RE-2016-375-338.

Cuenca, J.M. y López-Cruz, I. (2014). La enseñanza del patrimonio en los libros de texto de Ciencias Sociales, Geografía e Historia. Cultura y Educación. Revista de teoría, investigación y práctica, 26(1), 1-43. DOI: 10.1080/11356405.2014.908663

Dávila Acedo, M.A., Borrachero Cortés, A.B., Mellado Jiménez, V., y Bermejo García, M.L. (2015). Las emociones en alumnos de ESO en el aprendizaje de contenidos en física y química, según el género. International Journal of Developmental and Educational Psychology, 1(1), 173-180. DOI: 10.17060/ijodaep.2015.n1.v1.26

Earl, S.R., Taylor, I.M., Meijen, C. y Passfield, L. (2017). Autonomy and competence frustration in young adolescent classrooms: different associations with active and passive disengagement. Learning and Instruction, 49, 32-40. DOI: 10.1016/j.learninstruc.2016.12.001

Estepa, J. (2007). Investigando las sociedades actuales e históricas. Proyecto Curricular Investigando Nuestro Mundo (6-12). Sevilla: Díada.

Estepa-Giménez, J. (2013) (Ed.). La Educación patrimonial en la escuela y el museo: Investigación y experiencias. Huelva: Servicios de publicaciones de la Universidad de Huelva

Estepa Giménez, J., Wamba Aguado, A.M. y Jiménez Pérez, R. (2005). Fundamentos para una enseñanza y difusión del patrimonio desde una perspectiva integradora de las ciencias sociales y experimentales. Investigación en la Escuela, 56, 19-26.

Etxeberria, A.I., Merillas, O.F. y López, J.M.C. (2015). Actualidad y tendencias en Educación Patrimonial/Current issues and tendencies in Heritage Education. Educatio Siglo XXI, 33(1), 11-14.

Fernandes, I.M.B., Pires, D. y Delgado-Iglesias, J. (2018). ¿Qué mejoras se han alcanzado respecto a la Educación Científica desde el enfoque Ciencia-Tecnología-Sociedad-Ambiente en el nuevo Currículo Oficial de la LOMCE de $5^{\circ}$ y $6^{\circ}$ curso de Primaria en España? Revista Eureka sobre Enseñanza y Divulgación de las Ciencias, 15(1), 1101-1116. DOI: 10.25267/Rev_Eureka_ensen_divulg_cienc.2018.v15.i1.1101

Hart Dávalos, A. (2003). La teoría de la identidad Cultural y la Gobalización. Filosofía y Sociedad II. La Habana: Félix Varela

Hernández, M.I., Couso, D. y Pintó, R. (2015). Analyzing students' learning progressions throughout a teaching sequence on acoustic properties of materials with a model-based inquiry approach. Journal of Science Education and Technology, 24(2-3), 356-377. DOI: 10.1007/s10956-0149503-y

Hernández, A.M. y Guillén, R. (2017). La educación patrimonial en los manuales escolares de educación primaria: un recorrido desde LOGSE hasta LOMCE. Didáctica de las Ciencias Experimentales y Sociales, 32(2), 25-49. DOI: 10.7203/DCES.32.9205

Hodson, D. (2014). Nature of Science in the Science Curriculum: Origin, Development, Implication and Shifting Emphases. In M. Matthews (Ed.). International handbook of research in history, philosophy and science teaching (pp. 911-970). Dordrecht: Springer

Jiménez-Pérez, R., Cuenca, J.M., y Ferreras, M. (2010). Heritage education: exploring the conceptions of teachers and administrators from the perspective of experimental and social science teaching. Teaching and Teacher Education, 26(6), 1319-1331.

Khishfe, R., Alshaya, F.S., BouJaoude, S., Mansour, N. y Alrudiyan, K.I. (2017). Students' understandings of nature of science and their arguments in the context of four socio-scientific issues. International Journal of Science Education, 39(3), 299-334. DOI: 10.1080/09500693.2017.1280741 
Lacour, S. (2008). La complexité de la production d'information dans une démarche d'intelligence territoriale dans un secteur où les sources sont pluridisciplinaires et les informations multisectorielles. L'exemple de l'animation du patrimoine. En 6th annual international conference of Territorial Intelligence "Tools and methods of Territorial Intelligence", 1-8. Recuperado de https://halshs.archives-ouvertes.fr/halshs-00982194 [24 de mayo de 2019].

Lederman, N.G. (2018) La siempre cambiante contextualización de la naturaleza de las ciencias: documentos recientes sobre la reforma de la educación científica en los Estados Unidos y su impacto en el logro de la alfabetización científica. Enseñanza de las Ciencias, 36(2), 5-22.

López-González, L. y Oriol, X. (2016). La relación entre competencia emocional, clima de aula y rendimiento académico en estudiantes de secundaria. Cultura y Educación, 28(1), 130-156. DOI: $10.1080 / 11356405.2015 .1120448$

Lorusso, S., Cogo, G.M., y Natali, A. (2017). The protection and valorization of cultural and environmental heritage in the development process of the territory. Conservation Science in Cultural Heritage, 16(1), 59-88. DOI: 10.6092/issn.1973-9494/7165

Manassero, M.A. Vázquez, A. y Acevedo, J.A. (2002). Opiniones sobre la influencia de la ciencia en la cultura. Didáctica de las Ciencias Experimentales y Sociales, 16, 35-55

Matas, A. (2018). Diseño del formato de escalas tipo Likert: un estado de la cuestión. Revista Electrónica de Investigación Educativa, 20(1), 38-47. DOI: 10.24320/redie.2018.20.1.1347

Mattozzi, I. (2001). La didáctica de los bienes culturales. A la búsqueda de una definición. En J. Estepa, C. Domínguez y J. M. Cuenca (eds.). Museo y Patrimonio en la didáctica de las Ciencias Sociales (pp. 57-96) Huelva: Universidad de Huelva.

MECD (Ministerio de Educación, Cultura y Deporte) (2015). Real Decreto 1105/2014, de 26 de diciembre, por el que se establece el currículo básico de la Educación Secundaria Obligatoria y del Bachillerato. BOE, 37, 169-546.

Mellado, V., Borrachero, A.B., Brígido, M., Melo, L.V., Dávila, M.A., Cañada, F., Conde, M.C., Costillo, E., Cubero, J., Esteban, R., Martínez, G., Ruiz, C., Sánchez, J., Garritz, A., Mellado, L., Vázquez-Bernal, B., Jiménez-Pérez, R. y Bermejo, M.L. (2014). Las emociones en la enseñanza de las ciencias. Enseñanza de las Ciencias, 32(3), 11-36.

Merchán-Galán, A. (2018). Propuesta para promover la Alfabetización Científica en alumnos de $2^{\circ}$ de ESO mediante actividades Ciencia-Tecnología-Sociedad (Tesis doctoral). Universidad Internacional de La Rioja (UNIR). Logroño.

Moraga, S., Espinet, M. y Merino, C. (2019). El contexto en la enseñanza de la química: análisis de secuencias de enseñanza y aprendizaje diseñada por profesores de ciencias en formación inicial. Revista Eureka sobre Enseñanza y Divulgación de las Ciencias, 16(1), 1604. DOI: 10.25267/Rev Eureka ensen divulg cienc.2019.v16.i1.1604

Osborne, J, Simon, $\bar{S}$. y Collins, S (2003). Attitudes towars science: a review of the literatura and its implications. International Journal of Science Education, 25(9), 1049-1079. DOI: 10.1080/0950069032000012199.

Peñalva-Vélez, A., López-Goñi, J.J. y García-Manso, M.I. (2016). El desarrollo de las competencias emocionales en alumnado de secundaria: perfiles diferenciales en función del sexo. Educatio Siglo XXI, 34(1 Marzo), 223-242. DOI: 10.6018/j/253301

Piedra Sarría, Y.L. (2011). La Identidad Patrimonial, un Derecho Cultural reflejado en la Política Cultural Cubana a través del Programa Nacional de Patrimonio. En Contribuciones a las Ciencias Sociales. Recuperado de www.eumed.net/rev/cccss/12.

Planeta Isla (2018). Museum Molino Zaporito. Guía didáctica 1er. Ciclo Secundaria. Disponible en http://www.planetaisla.es/zona-de-descarga/

Porlán, R. (1990) Hacia una fundamentación espistemológica de las ciencias. Investigación en la Escuela, 10, 3-22. DOI:10.12795/IE.1990.110.01

Retana-Alvarado D.A., de las Heras Pérez M.A., Vázquez-Bernal B. y Jiménez-Pérez R. (2018). El cambio en las emociones de maestros en formación inicial hacia el clima de aula en una 
intervención basada en investigación escolar. Revista Eureka sobre ECDC, 15(2), 2602. DOI: 10.25267/Rev_Eureka_ensen_divulg_cienc.2018.v15.i2.2602

Retana-Alvarado, D.A. Vázquez-Bernal, B. y Camacho, M.M. (2018). Las Ferias de Ciencia y Tecnología de Costa Rica y sus aportes a la educación secundaria. Revista Electrónica Actualidades Investigativas en Educación, 18(2), 1-43. DOI: 10.15517/aie.v18i2.33170 ISSN 1409-4703.

Rite, M.T. y López, J.M.C. (2017). La educación patrimonial para la adquisición de competencias emocionales y territoriales del alumnado de enseñanza secundaria. PULSO. Revista de Educación, 40, 159-174.

Robinson, J.P., Shaver, P.R. y Wrightsman, L.S. (1991). Measures of personality and social psychological attitudes. San Diego, CA: Academic Press.

Rovetta, A., Rovida, E., y Zafferri, G. (2017). Heritage by Dynamic Museums Project with New Artificial Intelligence Suggestions. In Marco Ceccarelli, Michela Cigola y Giuseppe Recinto (Eds.) New Activities For Cultural Heritage (pp. 239-246). Switzerland: Springer.

Sánchez Mora, M.C. (2013). Museos de ciencias, escuelas y profesorado, una relación a revisarse. Revista Eureka sobre Enseñanza y Divulgación de las Ciencias, 10(3), 377-393. DOI: 10.25267/Rev_Eureka_ensen_divulg_cienc.2013.v10.i3.05

Santacana, J. (2007). Museografía didáctica, museos y centros de interpretación del patrimonio. En J. Santacana y N. Serrat (coords.), Museografía didáctica. Barcelona: Ariel, 395-471.

Scarantino. A. (2018). The philosophy of emotions and its impact on affective science. In L. Feldman Barrett, P. M. Niedenthal, y P. Winkielman (Eds.) Handbook of Emotions, 4th Ed. (pp. 22-104). New York: The Guilford Press.

Schneider, B., Krajcik, J., Lavonen, J., Salmela-Aro, K., Broda, M., Spicer, J., Bruner, J., Moeller, J., Linnsaari, J. y Viljaranta, J. (2016). Investigating optimal learning moments in US and Finnish science classes. Journal of Research in Science Teaching, 53(3), 400-421. DOI: $10.1002 /$ tea.21306

Solbes, J. (2013). Contribución de las cuestiones sociocientíficas al desarrollo del pensamiento crítico (I): Introducción. Revista Eureka sobre Enseñanza y Divulgación de las Ciencias, 10(1), 1-10. DOI: 10.25267/Rev_Eureka_ensen_divulg_cienc.2013.v10.i1.01

Strati, A.D., Schmidt, J.A. y Maier, K.S. (2017). Perceived challenge, teacher support, and teacher obstruction as predictors of student engagement. Journal of Educational Psychology, 109(1), 131-147. DOI: 10.1037/edu0000108

Strieder, R.B., Torija, B.B. y Quílez, M.J.G. (2017). Ciencia-tecnología-sociedad: ¿Qué estamos haciendo en el ámbito de la investigación en educación en ciencias? Enseñanza de las ciencias: revista de investigación y experiencias didácticas, 35(3), 29-49. DOI: $10.5565 / \mathrm{rev} / \mathrm{ensciencias} .2232$

Tobin, K. (2010). Reproducir y transformar la Didáctica de las Ciencias en un ambiente colaborativo. Enseñanza de las Ciencias, 28(3), 301-314.

UNESCO-Montevideo (1999). Declaración de Santo Domingo. La ciencia para el siglo XXI: una nueva visión y un marco de acción. Santo Domingo, República Dominicana, Recuperado de http://www.campus-oei.org/salactsi!santo-domingo.htm

\section{CÓMO CITAR ESTE ARTÍCULO}

Vázquez, B., De las Heras, M. A. y Jiménez-Pérez, R. (2020). Identidad patrimonial, emociones y enseñanza de las Ciencias Experimentales. Didáctica de las ciencias experimentales y sociales, 38, 153-170. DOI: 10.7203/DCES.38.15688. 
Anexo I.

\begin{tabular}{ll}
\hline \multicolumn{2}{l}{ Materia Física y Química de $2^{\circ}$ ESO. Fuentes: } \\
CEJA (2016a; 2016b); MECD (2015). \\
\hline Contenido & Bloque 2, punto 2.6: Métodos de separación de mezclas. \\
\hline \multirow{2}{*}{ Objetivo } & $\begin{array}{l}\text { 8. Conocer y valorar las interacciones de la ciencia y la tecnología } \\
\text { con la sociedad y el medio ambiente, para así avanzar hacia un } \\
\text { futuro sostenible. }\end{array}$ \\
\hline Criterios de evaluación & $\begin{array}{l}\text { 5. Proponer métodos de separación de los componentes de una } \\
\text { mezcla. }\end{array}$ \\
\hline Competencias & Lingǘstica, Ciencias, Cultural y Aprender a aprender. \\
\hline \multirow{2}{*}{ Estándar de aprendizaje } & $\begin{array}{l}\text { E.A.4.5.1. Diseña métodos de separación de mezclas según las } \\
\text { propiedades características de las sustancias que las componen, } \\
\text { describiendo el material de laboratorio adecuado. }\end{array}$ \\
\hline & "Planeta Isla Museum Molino Zaporito" dentro del proyecto \\
global "Ciencia Divertida". Metodología: la experimentación, \\
dramatización teatral y el uso de medios tecnológicos para \\
estimular el aprendizaje. Objetos generales: conocer aspectos \\
fundamentales de la cultura, promover el respecto a nuestro \\
patrimonio, conocer y aplicar métodos para identificar problemas, \\
fortalecer las capacidades afectivas del alumnado. Objetivos \\
específicos: conocer las principales características de los molinos \\
de mareas de la Bahía de Cádiz; conocer los acontecimientos \\
históricos que culminaron en la construcción del molino Zaporito; \\
analizar en una maqueta las principales partes de un molino \\
Planeta Isla (2018).
\end{tabular}

Fase de construcción escolar previo:

Actividades de aula relativas al contenido escolar "Métodos de separación se mezclas", con trabajo de lápiz, papel y material de laboratorio para familiarizar al alumnado con estos procesos físico-químicos: filtración, decantación, cromatografía, destilación, cristalización, centrifugación.

Fase de iniciación, planificación y búsqueda de información: Actividad 1 $1^{\text {a }}$ : La extracción de productos para la alimentación a partir de compuestos vegetales ha sido fundamental para el desarrollo de las sociedades y una constante en la historia de la humanidad. Especial mención merece el trigo, cuyo cultivo condujo a la formación de sociedades cada vez más complejas. Busca información sobre esta relación y resume la información y

La visita al Molino de mareas (desde una perspectiva indagatoria) cómo ha cambiado el proceso de extracción de la harina.

Actividad 2a: ¿Qué proceso seguirías para extraer la parte esencial y nutritiva del grano (harina) de la cáscara.

Actividad 3a: Reproduce el procesado con el material que te proporciona el profesor y explica qué sensaciones te has producido. ¿Cómo se denomina este proceso habitualmente? ¿Y su nombre científico?

Actividad $4^{\text {a }}$ : Imagina que deberías hacerlo, pero con grandes cantidades de grano, ¿a qué procesos crees que deberías someterse?

Actividad 5a: ¿Conoces algún ingenio en tu localidad o cerca de ella que se usara para reproducir este proceso en grandes cantidades?

Actividad 6: ¿Cómo crees que era su funcionamiento? ¿Se puede clasificar la máquina en algún tipo existente a lo estudiado?

Fases de estructuración y evaluación: 
Actividad $7^{\mathrm{a}}$ : En función de lo aprendido, ¿puedes describirlo? ¿En qué se parece a lo realizado en el laboratorio y en qué se diferencia? ¿Quién proporciona básicamente la energía? ¿Sabes qué está detrás de este fenómeno? ¿Supón que a las 12 horas del día está la pleamar, teóricamente cuántas veces podrías reproducirse al día este proceso de molturar el trigo?

Actividad $8^{\text {a }}$ : ¿Qué sensaciones te ha producido ver el molino de mareas del Zaporito?

Actividad 9a: ¿Puedes identificar los elementos que formaban el molino de mareas del río Arillo?

Actividad $10^{\mathrm{a}}$ : ¿Escribe sobre la maqueta del molino del Zaporito sus elementos constituyentes? ¿Reconoces algunos de sus elementos constituyentes?

Actividad $11^{\mathrm{a}}:$ ¿Qué acciones propondrías para ayudar a recuperar el molino del río Arillo?

Actividad 12a: ¿Conoces otros productos vegetales a los que aplicar este proceso de molturación?

Actividad 13a: Escribe un relato sobre tu experiencia en la actividad.

Fuente: Elaboración propia. 
Anexo II. Imagen del Molino de Mareas Zaporito restaurado (San Fernando).

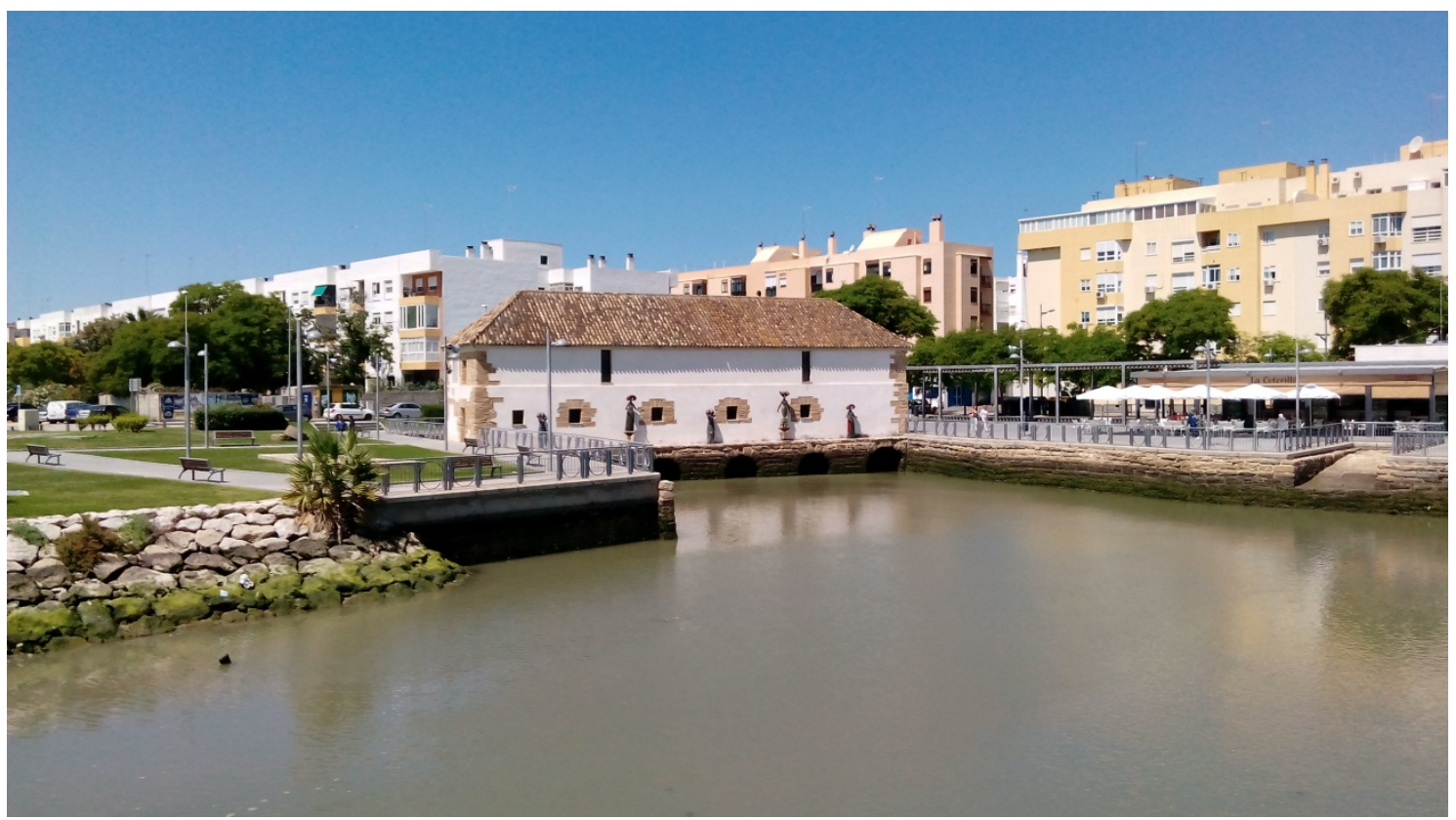

Fuente: Elaboración propia

Anexo III. Imagen del Molino de Mareas Río Arillo ruinoso (Cádiz-San Fernando).

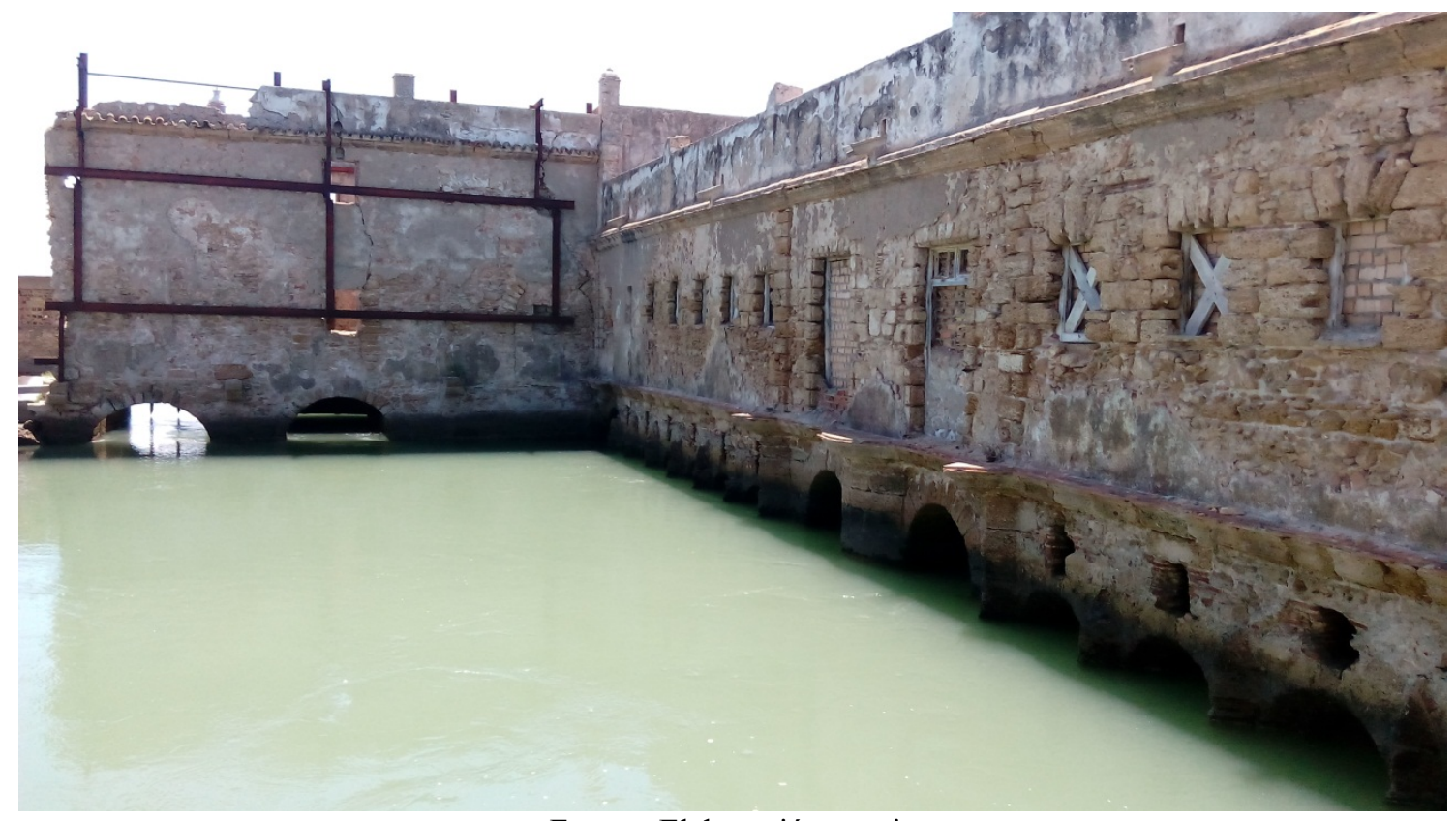

Fuente: Elaboración propia 